\title{
Vision-Based Markerless Gaming Interface
}

\author{
Pietro Azzari and Luigi Di Stefano \\ ARCES - DEIS, University of Bologna, \\ viale Risorgimento 2, 40125 Bologna, Italy \\ pazzari@unibo.it \\ http://www.vision.deis.unibo.it/
}

\begin{abstract}
The paper proposes a novel human machine interface for gaming applications based on computer vision. The key idea is to allow the user to interact with the game by simply moving a hand-held consumer grade camera. Detection of natural features in the incoming video stream avoids instrumenting the scene with optical markers while preserving real-time computation and accuracy. The paper presents also a prototype videogame developed as proof-of-concept of our camera-based gaming interface. Thanks to recent advances in real-time extraction and matching of natural features from images on mobile platforms, our proposal holds the potential to enable a new generation of camera-controlled videogames for hand-held mobile devices.
\end{abstract}

Keywords: human-machine interfaces, camera pose estimation, videogames, keypoint matching.

\section{Introduction}

The ever increasing pervasiveness of computer systems into our everyday environment calls for novel mechanisms of human-computer interaction. Interfaces to computerized equipment need to be straightforward and effective, the ability to interact using inexpensive tools being highly regarded.

In the last decades, keyboard and mouse have become the main interfaces for transferring information and commands to computerized equipment. In some applications involving 3D information, such as visualization, computer games and control of robots, other interfaces based on remote controller [16, joysticks and wands can improve the communication capabilities despite being sometimes impractical or limited.

Wearable and handheld devices, such as datagloves, "backpacks" [3] and haptics, are designed to be more user friendly, helping untrained users in performing complex tasks. On the other hand, the high cost and cumbersome hardware limit the field of usability of these solutions.

In daily life, however, vision and hearing are the main channels through which humans gather information about their surroundings. Therefore, the design of new interfaces that allow computerized equipment to communicate with humans by understanding visual and auditive input may conjugate effectiveness, naturalness and affordable prices. 
Vision based interfaces hold the potential to communicate with computerized equipment at a distance and the machine can be taught to recognize and react to human-like feedbacks. Despite many advances have been recently reached in the field of human gesture, motion and behaviour understanding [10 2011], engineers have been mostly focusing on marker-based tracking systems for humancomputer interaction applications. The gaming industry is recently showing a deep interest in vision based interfaces, with many proof of concepts developed so far $7 / 212$ 217]. As a matter of fact visual markers can be reliably tracked [5] at low computational costs, nonetheless game boards or game controllers must be instrumented with them.

Conversely, this paper presents a novel vision-based gaming interface able to deliver position and orientation of the player by simply using a hand-held consumer grade camera and without requiring any visual marker. The proposed approach is straightforward since the movement of the camera directly translates into 3D commands to the game and requires no instrumentation of the environment. It is also very effective since camera pose is estimated with millimetric precision. Finally it is cheap since it relies on widely available low-cost cameras.

\section{Related Work}

Recent works in literature show that, to some extent, human behaviour understanding using imaging devices is attainable. Harville and al. 10] conceived a robust algorithm for 3D person tracking and activity recognition. The work by Viola and Jones [20] paved the way for sound automatic face detection. Isard and al. 11] demonstrated reliable tracking of deformable objects in presence of occlusion and cluttered environments. These outstanding achievements have inspired the works of $\mathrm{Lu}$ [15 14] on vision-based game interfaces controlled respectively by head and hands movements. Head, face and body position tracking for computer games was also successfully demonstrated in the work of Freeman et al. 6]. However, despite being very flexible and natural interfaces from a human perspective, the underlying technology is still too computational intensive to guarantee short latency time and smooth operations. Moreover precise handling and maneuvering tasks demand a detection and reconstruction accuracy that, in some cases, current algorithms may not deliver.

Tracking of optical markers has rapidly emerged as a fast and accurate alternative for conveying simplified information to computer systems. Although complex human behaviours cannot be captured, location and orientation information can be robustly retrieved in a wide variety of environmental conditions and at low computational cost. Examples of videogames built on top of optical marker trackers have been growing steadily in recent years. Cho et al. 2] described an augmented reality shoot-em-up game in which players aim at virtual opponents rendered on a game board filled with optical markers. Oda et al. 17] developed a racing game where users steer their virtual cars using controllers stuck with markers monitored with cameras. Govil and al. 7] designed a marker-based golf ball tracker used to set speed and direction of a virtual ball in a golf simulator. 
By exploiting implementation of a markers tracker for portable devices, Wagner and colleagues [21] developed an Augmented Reality (AR) game where multiple players are allowed to interact using camera-equipped PDA devices.

Nonetheless, recent advances in the field of object recognition showed that accurate pose estimation and tracking can be achieved without the need of specific visual markers, but instead using keypoints extracted from textured areas 13. In particular, the SURF (Speeded Up Robust Features) algorithm [1] reconciled accuracy and low computational cost for robust keypoints extraction and tracking.

In this paper we originally propose to deploy a camera pose estimation approach based on natural keypoints correspondences as a human-machine interface for gaming purposes. It is worth pointing out that camera pose estimation using natural keypoints on mobile phones has been recently demonstrated by Wagner et al. 22. Hence, our proposal holds the potential for development of new camera-controlled gaming applications for hand-held mobile devices such as phones and PDAs. In the remainder of the paper we describe the camera pose estimation algorithm in terms of its key components and present a prototype videogame, dubbed Black Hole, developed so far as proof-of-concept of our proposed approach.

\section{Markerless Pose Estimation}

The interface consists essentially of an automatic camera pose estimation algorithm for scenes in which flat objects are present, therefore limiting the types of suitable scenes. In this case, however, the limitation is slight, since the requirements is that a plane be visible, even if partially occluded, in the scene. This is common in indoor environments, where a textured ceiling or ground plane is usually visible. Outdoors, even rough ground (grass, roads or pavements), provides also an acceptable reference for the system.

The pose recovery algorithm is largely inspired by the camera tracker illustrated by Simon et al. [19, for it delivers accurate estimation at low computational cost. However, differently from the original formulation, pose recovery is performed every time with respect to a reference frame (pose detection) instead of arising from the composition of multiple pairwise registration (pose tracking) among subsequent frames. Hence, pose detection tolerates failures since each frame is processed independently; besides it does not suffer from the dead reckoning issue typical of pairwise composition. On the other hand, pose detection requires a reference object to be known beforehand, i.e. the object with respect to which the pose is continuously computed. Moreover, pose jittering may arise since temporal correlation is usually not reinforced. In the rest of this section the solutions to these two problems are addressed and described.

Using natural keypoints instead of markers makes the instrumentation of the scene not needed anymore since any flat object can be a suitable reference. Just before starting a gaming session a brand new natural reference can be learnt on-the-fly by simply taking a snapshot of a textured planar object and 
extracting a vector of keypoints descriptors. The corresponding points of the reference keypoint set are searched within every new incoming frame and pairs of matching keypoints are likely to be detected even in case of large pose and illuminations changes, as shown in Fig. 1. Incorrect keypoints pairs can be easily detected and discarded using a RANSAC-based homography estimation step 9. The remaining correspondences are fed to the pose estimation algorithm described in [19] in order to obtain a reliable estimation of the position and orientation of the camera with respect to the reference object.

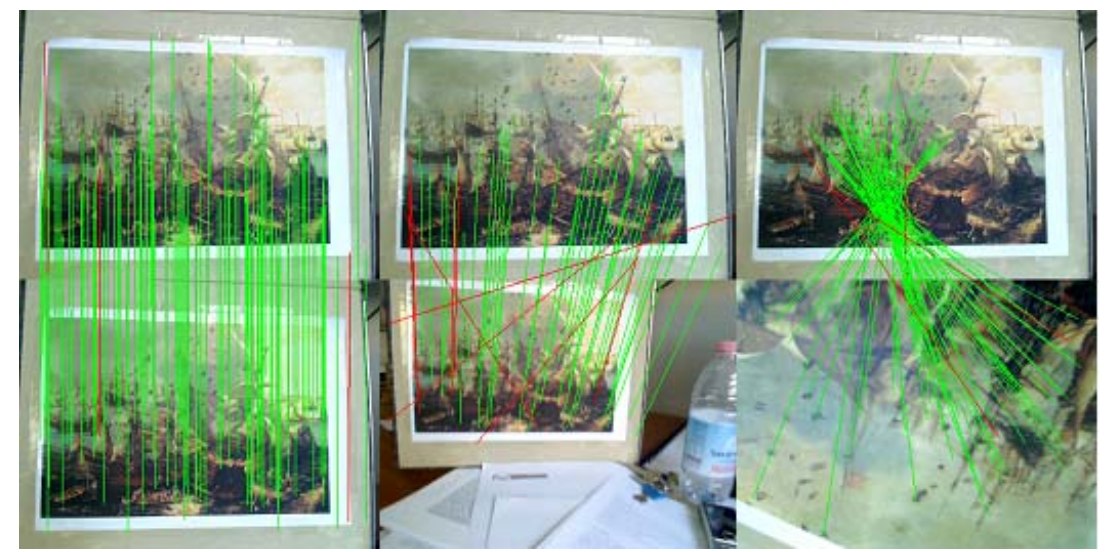

Fig. 1. Tracking SURF keypoints in few snapshots taken from different viewpoints: correct (green) and incorrect (red) corresponding pairs

The way the pose is obtained is depicted in Fig. 2, keypoints $m_{i}$, located on the camera imaging sensor (bottom left plane), are in one-to-one correspondence with points $M_{i}$ standing on a flat reference object (upper right plane). It can be assumed, without loss of generality, that the reference object lays on the $z=0$ plane of the world coordinate frame, so that $2 \mathrm{D}$ points $M_{i}$ can be augmented with a third null coordinate. The set of corresponding 2D-3D points $\left(m_{i}, M_{i}\right)$, of which $\tilde{m}, \tilde{M}$ are just the homogeneous notations, are related by projective equations involving the internal camera matrix $\mathrm{A}$, the rotation matrix $R$ and the translation vector $t$

$$
s \tilde{m}=A[R t] \tilde{M}
$$

Both $R$ and $t$ can be retrieved up to a scalar value $s$, by means of the method described in [19], provided that enough corresponding pairs $\left(m_{i}, M_{i}\right)$ are available and the camera is internally calibrated.

Independent pose estimation computed at every frame exhibit an excellent accuracy with camera position usually estimated in the range of few millimeters from the true one. Nonetheless, since this approach does not exploit the temporal continuity of the camera trajectory, the sequence of estimated poses usually exhibit jitter effects. This problem manifests as small vibrations among 


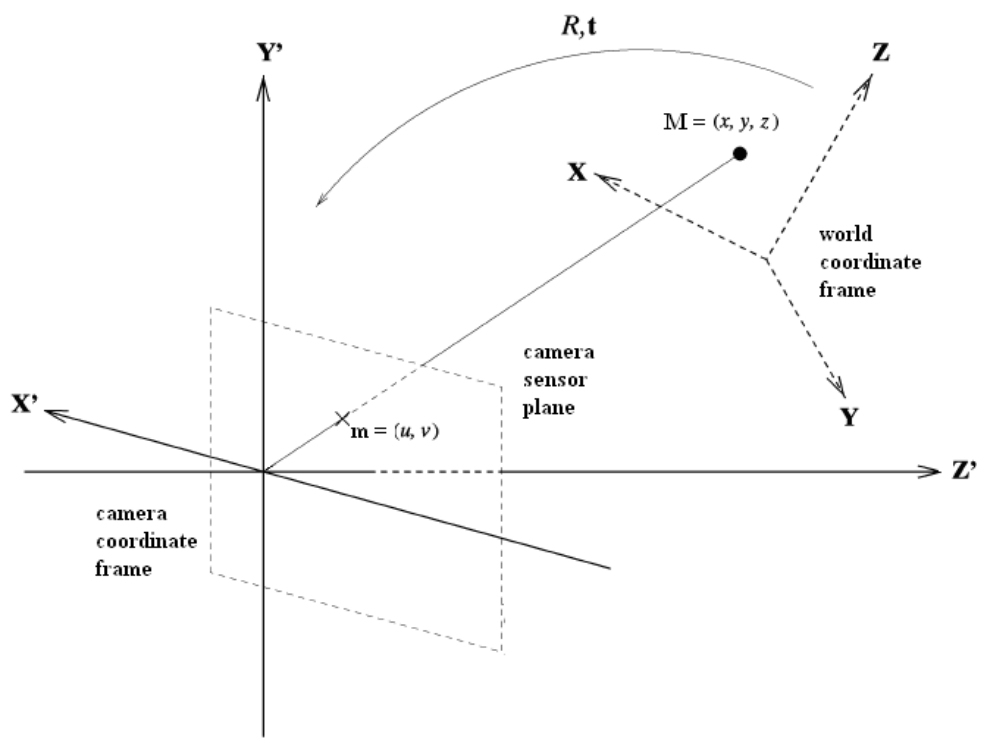

Fig. 2. Geometry of pose estimation from 2D-3D correspondences problem

subsequent estimations, such discontinuities being quite noticeable by a human observer and tending to degrade the gaming experience. In order to mitigate this effect a pose smoothing technique has been adopted. The developed approach, described in [18, consists in linking every new pose with those computed during a previous time window by exploiting a Support Vector Regression scheme as a temporal regularization term.

Natural keypoints correspondences and pose smoothing make the conceived pose estimation algorithm fast, robust and practical, thus providing accurate and jitter-free estimations without the need for fiducial markers placed all across the scene.

\section{Game Sessions}

A prototype videogames has been developed using as interface the vision-based pose estimation algorithm described previously. In addition, we integrated few third-party libraries for a number of specialized tasks, in particular:

- OpenGL (Open Graphics Library) [8], a portable and interactive 2D and 3D graphics library adopted for fast visualization and rendering.

- OpenCV (Open Computer Vision) [4, a collection of computer vision functions used for video capturing, keypoints detection and numerical optimization.

- Tokamak [12], an open-source real-time physics engine used for accurate simulation of dynamics of rigid body, gravity, friction ans so on. 

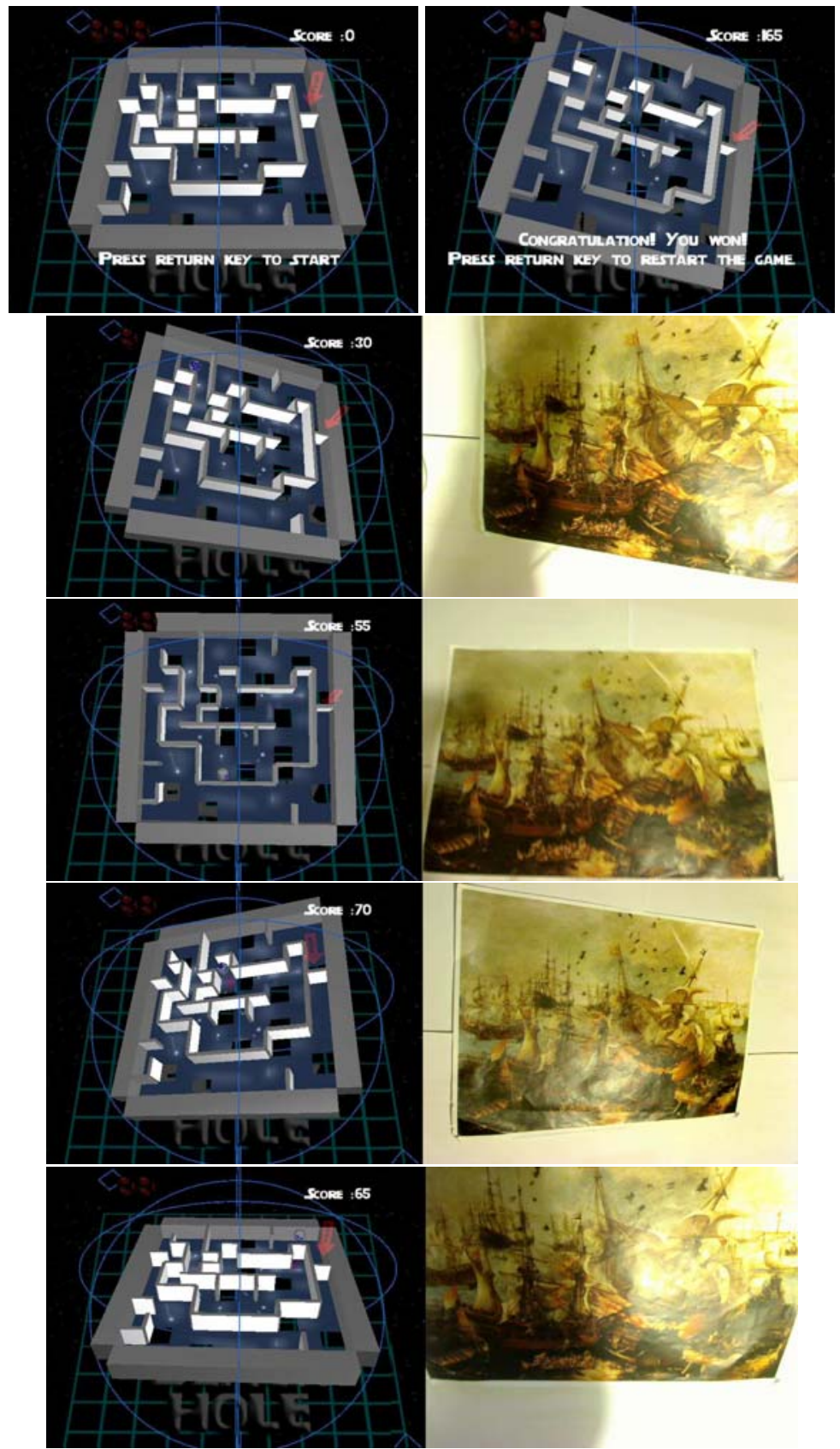

Fig. 3. Black Hole starting screen (top left) and game ending (top right). Below the snapshots depict the floating maze (left) tilting and panning according to the orientation of the camera with respect to the reference object (right). 
The typical hardware configuration used to run the game consists of a single laptop pc powered by an Intel Core 2 CPU, equipped with 4 GB RAM and running Windows XP. The used video camera is a Logitech QuickCam Sphere grabbing color sequences at $640 \times 480$ resolution. The game has been developed in $\mathrm{C}++$ using Microsoft Visual Studio 2005. Using this setting the frame rate ranges between 6 and 10 frames per second (FPS), keypoints extraction being the major bottleneck of the system. Although quite far from real-time processing, the system is responsive enough to allow for a satisfactory gaming experience. By reducing the camera resolution to $320 \times 240$ the frame rate increase to $9-15$ FPS without severely penalizing the accuracy.

\subsection{Black Hole}

Black Hole is a puzzle game inspired by the dark atmosphere of Star Wars. The goal is to steer a R2D2-like ball through a Death Star maze till the endpoint avoiding the holes spread along the path. The user can slant and rotate the maze by moving a webcam held in his hand. Gravity effect allows the user to control the ball by moving the maze; friction and collision against maze walls and floor are also implemented in order to add realism. Every time the user loses a ball by letting it fall in a hole it obtains a number of points commensurate to the distance from the starting point. After three lost balls the game ends and the final score is the sum of the points obtained thus far.

Fig. 3 shows the starting and ending screens of Black Hole together with some screenshots taken during a gaming session. The Figure includes also the images coming from the webcam hand-held by the player in correspondence of each screenshot, the reference object being a textured picture printed on a paper sheet and laying on the desktop. The corresponding pairs screenshot-camera image show how the floating maze is tilted according to the instantaneous orientation of the hand-held camera with respect to the reference object.

\subsection{Feedback and Observations}

The game has been on show for few weeks in our laboratory rooms and has been played by some colleagues from other labs that gently provided feedbacks and suggestions. First of all, only a picture of a person pointing the camera to the reference pattern laying on the table, has been required by anybody to start playing the games. Such a limited amount of training information hints at the ease of use and naturalness of the conceived interface. Most of the players manage to get to the end of the game, this suggesting also good intuitiveness and friendliness. On the other hand several persons expressed concerns about the difficulty of keeping the reference object always in sight during the gaming session. Even though occasional pose estimation failure does not necessarily muck up the game, it can be ennoying especially during agitated phases. Another set of complains concerns the responsiveness of the gameplay which is mainly accountable to the high computational cost that the system incur when highly textured areas generate a large amount of keypoints. 


\section{Conclusions and Future Work}

The ubiquitous presence of computerized equipments in everyday environment calls for conception and design of easy-to-use and natural human-machine interfaces. Practical, straightforward and inexpensive are the keywords for the next generation of interaction paradigms. Videogames are a challenging test ground since fast response and high accuracy are also required. Vision-based interfaces hold the potential to fulfill this expectation. In this paper a vision-based approach based on tracking natural features has been conceived as an interface for gaming applications. The proposed approach allows the user to interact with a videogame by simply moving a webcam pointing towards some planar textured object present in the scene. According to the feedback received by several users, the interface is intuitive, fast, responsive and, ultimately, enjoyable.

As for future directions of works, pose estimation from non-flat surfaces or larger-than-a-single frame object would prove useful to increase the possibility for the user to move around. Moreover, as for the difficulty of keeping the reference object always in sight, we wish to investigate on the possibility of enabling also a mixed-reality mode, in which the user would see the virtual objects of the game superimposed to actual video stream coming from the camera.

Eventually, the proposed approach is particularly suited to enable gaming applications on hand held devices such as phones and PDA, for the user may simply point the camera toward a textured plane and play by moving the device in his hand. Therefore, in the near feature we plan to port our gamimg interface on a state-of-the-art hand held device.

\section{Acknoledgements}

We would like to thank Marisa Panaccio for the development of the games, during her MS degree thesis, and for providing us with the images shown in Fig. 3 ,

\section{References}

1. Bay, H., Ess, A., Tuytelaars, T., Van Gool, L.: Surf: Speeded up robust features. Computer Vision and Image Understanding 110(3), 346-359 (2008)

2. Cho, K., Kang, W., Soh, J., Lee, J., Yang, H.S.: Ghost hunter: a handheld augmented reality game system with dynamic environment. In: Proc. of Intl. Conf. on Entertainment Computing, pp. 10-15 (2007)

3. Close, B., Donoghue, J., Squires, J., De Bondi, P., Morris, M., Piekarski, W., Thomas, B.: Arquake: an outdoor/indoor augmented reality first person application. In: Proc. of IEEE Intl. Symp. on Wearable Computers, pp. 139-146 (2000)

4. Intel Corp. Opencv 1.1, open computer vision library (2000-2008), http://www.intel.com/technology/computing/opencv/

5. Fiala, M.: Artag, a fiducial marker system using digital techniques. In: Proc. of IEEE Intl. Conf. on Computer Vision, pp. 590-596 (2005) 
6. Freeman, W.T., Tanaka, K., Ohta, J., Kyuma, K.: Computer vision for computer games. In: Proc. of Intl. Conf. on Automatic Face and Gesture Recognition, pp. 100-105 (1996)

7. Govil, A., You, S., Neumann, U.: A video-based augmented reality golf simulator. In: Proc. of ACM Multimedia, pp. 489-490 (2000)

8. Khronos Group. Opengl 2.1, open computer graphics library (1992-2008), http://www.opengl.org/

9. Hartley, R., Zisserman, A.: Multiple view Geometry in computer vision, 2nd edn. Cambridge University Press, Cambridge (2003)

10. Harville, M., Li, D.: Fast, integrated person tracking and activity recognition with plan-view templates from a single stereo camera. In: Proc. of Intl. Conf. on Computer Vision and Pattern Recognition, pp. 398-405 (2004)

11. Isard, M., Blake, A.: Condensation - conditional density propagation for visual tracking. Intl. Journal of Computer Vision 29(1), 5-28 (1998)

12. Lam, D.: Tokamak, open physics engine library, http://www.tokamakphysics.com/

13. Lowe, D.G.: Distinctive image features from scale-invariant keypoints. Intl. Journal of Computer Vision 60(2), 91-110 (2004)

14. Lu, P., Chen, Y., Zeng, X., Wang, Y.: A vision-based game control method. In: Proc. of Intl. Conf. on Computer Vision, Workshop on Human Machine Interaction, pp. 70-78 (2005)

15. Lu, P., Zeng, X.Y., Huang, X., Wang, Y.: Navigation in $3 \mathrm{~d}$ game by markov model based head pose estimating. In: Proc. of Intl. Conf. on Image and Graphics, pp. 493-496 (2004)

16. Nintendo(C. Wii, http://wii.nintendo.com/

17. Oda, O., Lister, L.J., White, S., Feiner, S.: Developing an augmented reality racing game. In: Proc. of Intl. Conf. on Intelligent Technologies for Interactive Environment (2008)

18. Salti, S., Di Stefano, L.: Svr-based jitter reduction for markerless augmented reality. In: Proc. of Intl. Conf. on Image Analysis and Processing (2008) (submitted paper)

19. Simon, G., Fitzgibbon, A.W., Zisserman, A.: Markerless tracking using planar structures in the scene. In: Proc. of Intl. Symposium on Augmented Reality, May-June 2000, pp. 120-128 (2000)

20. Viola, P., Jones, M.J.: Robust real-time face detection. Intl. Journal of Computer Vision 57(2), 137-154 (2004)

21. Wagner, D., Pintaric, T., Ledermann, F., Schmalstieg, D.: Towards massively multi-user augmented reality on handheld devices. In: Proc. of Intl. Conf. on Pervasive Computing, pp. 208-219 (2005)

22. Wagner, D., Reitmayr, G., Mulloni, A., Drummond, T., Schmalstieg, D.: Pose tracking from natural features on mobile phones. In: Proc. of Intl. Symp. on Mixed and Augmented Reality, pp. 125-134 (2008) 